\section{Activity of eribulin mesylate in heavily pretreated breast cancer granted access via the Cancer Drugs Fund}

\author{
Ramya Ramaswami ${ }^{1 \ddagger}$, Sean M O'Cathail ${ }^{1 \ddagger}$, James H Brindley ${ }^{1}$, Paul Silcocks ${ }^{2}$, \\ Sarah Mahmoud ${ }^{3}$ \& Carlo Palmieri ${ }^{* 1,4,5,6}$
}

\begin{abstract}
Aim: Eribulin mesylate is a synthetic analog of halichondrin B and is licensed for the treatment of patients with locally advanced or metastatic breast cancer that has progressed following treatment with anthracyclines and taxanes. It was not deemed to be cost effective based on a cost analysis by the National Institute for Health and Care Excellence in England and therefore it is not funded routinely by the National Health Service. The establishment of the Cancer Drugs Fund in England subsequently enabled access. As with any new chemotherapy drug that enters clinical practice for metastatic breast cancer (MBC) it is often used in heavily pretreated patients and the experience in a routine clinical setting can differ from that in a clinical study. We therefore present the experience of the first 25 cases treated at our institution via the Cancer Drugs Fund. Materials \& methods: A total of 25 patients were treated and in the 22 assessable cases the objective response rate was $18 \%$ (four out of 22), with a clinical benefit rate of $41.0 \%$ (9 out of 22). Results: The median time-to-progression and overall survival were 4.08 months and 5.89 months, respectively. There was a significant difference in clinical benefit rate (odds ratio: $0.065 ; 95 \% \mathrm{Cl}: 0-0.529$; $\mathrm{p}=0.0055$ ), as well as time-to-progression (hazard ratio: $9.18 ; 95 \% \mathrm{Cl}: 2.26-37.38 ; \mathrm{p}=0.002$ adjusted for age at diagnosis and interval between initial $\mathrm{MBC}$ diagnosis and commencing eribulin) favoring those patients who had not been rechallenged. There was no significant difference in overall survival (hazard ratio: $1.16 ; 95 \% \mathrm{Cl}: 0.44-3.05 ; \mathrm{p}=0.770$ adjusted for age at diagnosis and interval between initial diagnosis of $\mathrm{MBC}$ and commencing eribulin). Conclusion: Eribulin mesylate shows clinical activity; however, there appears to be differences in terms of benefit in patients based on whether patients have been rechallenged with an anthracycline and/or a taxane. These data require confirmation in larger patient groups.
\end{abstract}

Halichondrin B was the most potent of eight anti-tumor compounds derived from Halichondria okadai Kadota, a widely distributed black sea sponge found off the Pacific coast of Japan [1]. The initial cytotoxicity of the halichondrions and homohalicondrins was based on in vitro and in vivo studies of the melanoma cell line B-16 and leukemia cell lines P-388 and L-1210 [1]. Previously, okadaic acid, which is a potent inhibitor of serine/threonine PP1 and PP2A and itself cytotoxic, had been isolated from H. Okadai [2]. One of the major challenges in taking halichondrin B

\footnotetext{
'Department of Medical Oncology, Imperial College Healthcare NHS Trust, Fulham Palace Road, London, W6 8RF, UK

${ }^{2}$ Cancer Research UK Liverpool Cancer Trials Unit, Block C, Waterhouse Building, Liverpool, L69 3GL, UK

3Pharmacy Department, Imperial College Healthcare NHS Trust, Fulham Palace Road, London, W6 8RF, UK

${ }^{4}$ Department of Molecular \& Clinical Cancer Medicine, Institute of Translational Medicine, University of Liverpool,

Liverpool, L69 3GA, UK

${ }_{5}^{5}$ Liverpool \& Merseyside Academic Breast Unit, Royal Liverpool University Hospital, Liverpool, L7 8XP, UK

${ }^{6}$ Academic Department of Medical Oncology, Clatterbridge Cancer Center NHS Foundation Trust, Wirral, CH63 4JY, UK

*Author for correspondence: Tel.: +44 151706 4482; Fax: +44 151706 5826; c.palmieri@liverpool.ac.uk

${ }^{\ddagger}$ Authors contributed equally
}

\section{KEYWORDS}

- breast cancer $\bullet$ Cancer

Drugs Fund • eribulin

- metastatic • rechallenge 
forward as an anti-tumor drug was the very low amounts that could be derived from $H$. Okadai, with $600 \mathrm{~kg}$ of $H$. Okadai being required to produce the initial $12.5 \mathrm{mg}$ of halichondrin B, as well as its complex structure of 32 stereocenters [1]. However, the publication of a complete synthesis of halichondrin B [3], as well as the subsequent discovery that its biological activity resides in its macrocyclic lactone $\mathrm{C} 1-\mathrm{C} 38$ moiety [4] led to the development of more structurally simple, fully synthetic analogs that retain the biological activity of halichondrin B. The methodology for the synthesis of these analogs is patented by Eisai Pharmaceuticals (MA, USA) [5]. Over 180 such analogs were synthesized and tested, resulting in the identification of two macrocyclic ketone analogs of halichondrin B, named ER-076349 and ER-086526 [6], which were shown to have highly potent in vitro and in vivo anticancer activities.

In vitro studies of ER-076349 and ER-086526 demonstrated that they could inhibit growth at subnanomolar concentrations of several human cancer cell lines including breast cancer (MDA-MB-435), colon cancer (COLO 205 and DLD-1) and prostate cancer (LNCaP and DU 145) with greater potency than vinblastine and paclitaxel [6]. Marked in vivo activities at doses of $0.1-1 \mathrm{mg} / \mathrm{kg}$ were observed for these two halichondrin analogs against four human xenografts: MDA-MB-435 breast cancer, COLO 205 colon cancer, LOX melanoma and NIH:OVCAR-3 ovarian cancer. ER-086526 demonstrated superior efficacy at lower doses compared with paclitaxel [6], while in a paclitaxel-resistant human ovarian cancer cell line ER-086526 retained essentially complete in vitro activity [7].

ER-086526 (E7389 or NSC 707389) was taken forward for clinical development and later named as eribulin mesylate (herein referred to as eribulin). Eribulin binds to tubulin and interferes with microtubule dynamics. It has a novel mechanism of action in that it predominantly inhibits microtubule growth, but not shortening, resulting in sequestration of tubulin into nonfunctional aggregates [8]. This is unlike other antimicrotubule agents such as vinca alkaloids and taxanes that suppress both the shortening and growth phases of microtubule dynamics. Eribulin interference with microtubular function leads to blockade of the $\mathrm{G}_{2}-\mathrm{M}$ phase of the cell cycle, disruption of mitotic spindles and apoptosis of tumor cells $[6,8]$.

\section{Phase I studies}

Four Phase I studies have been performed. The first was performed with eribulin administered as a 1- to 2-min intravenous (iv.) bolus on days 1, 8 and 15 of a 28-day cycle at a dose of $0.125-2.0 \mathrm{mg} / \mathrm{m}^{2}$ [9]; the same schedule was subsequently used but with eribulin administered at a dose of $0.25-2.0 \mathrm{mg} / \mathrm{m}^{2}$ as a 1 -h infusion [10]. In the third study, eribulin was administered as a 1-h infusion every 21 days at a dose of $0.25-4 \mathrm{mg} / \mathrm{m}^{2}$ [11] and, following data from two initial Phase II studies [12,13], a fourth study with the eribulin administered over 2-10 min iv. on day 1 and 8 of a 21-day cycle at a dose of $0.7-2.0 \mathrm{mg} / \mathrm{m}^{2}$ was performed [14]. In all of these studies neutropenia and/or febrile neutropenia were the most commonly reported dose-limiting toxicities (DLT).

Eribulin on days 1, 8, and 15 of a 28-day cycle (1- to 2 -min iv. bolus) at a dose of $2.0 \mathrm{mg} / \mathrm{m}^{2}$ resulted in two DLTs (febrile neutropenia and neutropenia), and the maximum tolerated dose (MTD) was determined as $1.4 \mathrm{mg} / \mathrm{m}^{2}$ [9]. While eribulin, over an 1-h infusion according to the same treatment schedule as the first study at a dose of $1.4 \mathrm{mg} / \mathrm{m}^{2}$, resulted in two DLTs (two neutropenia and one of fatigue in one of these patients), in addition in three other patients neutropenia resulted in omission of cycle 1 day 15 treatment. The MTD in this study was $1.0 \mathrm{mg} / \mathrm{m}^{2}$ [10]. When administered every 21 days at a dose of $4 \mathrm{mg} / \mathrm{m}^{2}$, a DLT (febrile neutropenia) was experienced by three patients on day 7 [11]. At a reduced dose of $2.8 \mathrm{mg} / \mathrm{m}^{2}$ a further two patients experienced the same DLT, and the MTD was subsequently determined to be $2 \mathrm{mg} /$ $\mathrm{m}^{2}$ [11]. Eribulin given on days 1 and 8 every 21 days resulted in three DLTs (neutropenia) at $2.0 \mathrm{mg} / \mathrm{m}^{2}$, and the MTD was determined to be $1.4 \mathrm{mg} / \mathrm{m}^{2}$ [14]. At a dose of $1.4 \mathrm{mg} / \mathrm{mg}^{2}$ on days 1 and 8 every 21 days the median time from day 1 to nadir in neutrophil count was 14 days for eribulin $1.4 \mathrm{mg} / \mathrm{m}^{2}$ (range: 11-14 days), in patients who received only their day 1 dose [14]. The median time to recovery from the nadir to grades 0 or 1 was 8 days for patients receiving eribulin on both days 1 and 8 [14]. Observed nonhematological toxicities included hypoglycemia, hypophosphatemia and fatigue [9], peripheral neuropathy $[10,14]$, and elevated $\gamma$-glutamyltransferase (liver metastasis) [14]. Fatigue and alopecia were also reported across the studies.

Pharmacokinetic analysis demonstrated that eribulin displayed linear kinetics over the dose 
range studied, that it had a high volume of distribution, and a rapid distribution phase followed by a slow elimination phase [9-11,14]. Urinary excretion of eribulin is minimal with $5-13 \%$ of the administered dose eliminated in urine $[10,11,14]$. A subsequent study utilizing ${ }^{14} \mathrm{C}$-eribulin found that eribulin undergoes limited metabolism and confirmed the minimal excretion of eribulin in the urine. The predominant route of elimination is primarily in the feces [15].

Given that hepatic involvement in malignant disease is common and can affect hepatic function and, therefore, the handling and excretion of drugs, a Phase I study was also performed to determine the pharmacokinetics of eribulin in patients with solid tumors and hepatic impairment, with the objective of defining the safety of eribulin in such patients [16]. In total, 18 patients were recruited, six with normal hepatic function, seven with Child-Pugh A and five with Child-Pugh B group hepatic impairment. Eribulin was given on day 1 and 8 of a 21-day cycle at a dose of $1.4,1.1$ and $0.7 \mathrm{mg} / \mathrm{m}^{2}$ for normal, Child-Pugh A and Child-Pugh B patients, respectively. Although the dose could be escalated in those starting on lower doses this did not happen in any cases. Hepatic impairment was found to increase the exposure to eribulin, with the mean dose-normalized area under the curve increased by 1.75 -fold (90\% CI: 1.16-2.65) in those with Child-Pugh A and 2.48-fold (90\% CI: 1.57-3.92) in Child-Pugh B as compared with patients with normal hepatic function [16]. Hepatic impairment also decreased clearance and prolonged elimination half-life of eribulin. The underlying reason for the increased exposure with eribulin in patients with hepatic dysfunction is reduced biliary excretion, as a result of reduced biliary flow, as well as reduction in transporter-mediated excretion in the bile. This study supports routine dose reduction in patients with Child-Pugh A or B hepatic impairment who are to receive eribulin.

\section{Phase II studies}

Eribulin has been tested in a number of Phase II studies in locally advanced and metastatic breast cancer (MBC) [12,17-20], as well as a feasibility study in the adjuvant setting [21]. The initial Phase II studies explored the efficacy of eribulin in a heavily pretreated population that had received a median of three to four prior chemotherapy regimens $[12,17,18]$. Subsequently, it has been tested as first-line therapy for locally recurrent or MBC in both HER2-negative [19] and HER2-positive disease where it was combined with trastuzumab [20]. Table 1 summarizes these Phase II studies.

In the two initial Phase II studies, one in $\mathrm{MBC}$ and the other in non-small-cell lung cancer, a dose of $1.4 \mathrm{mg} / \mathrm{m}^{2}$ as a $2-5$-min iv. infusion on days 1,8 , and 15 of a 28-day cycle was selected $[12,13]$. However, $63 \%$ of patients ( 44 out of 70) experienced dose delays associated with neutropenia during the first cycle, resulting in omission of the day 15 dose. Therefore, the schedule was modified to day 1 and 8 of a 21-day cycle [12]. Similarly, in the non-smallcell lung cancer Phase II study, hematological toxicities resulted in dose interruptions, delays, or omissions when eribulin mesylate $1.4 \mathrm{mg} / \mathrm{m}^{2}$ was administered on days 1, 8 and 15 of a 28-day cycle. The dosing schedule was again changed to days 1 and 8 of a 21-day cycle [13]. Given that a formal Phase I study had not been undertaken to determine the optimal dose for eribulin mesylate when administered on days 1 and 8 of a 21-day cycle a fourth Phase I study was established [14]. This determined that $1.4 \mathrm{mg} / \mathrm{m}^{2}$ administered on days 1 and 8, of a 21-day cycle was the MTD; this was the dosing regimen taken forward in all subsequent studies.

\section{Feasibility study for adjuvant trial}

As well as these Phase II studies a feasibility study is currently examining eribulin as adjuvant therapy following dose-dense doxorubicin (A) and cyclophosphamide (C) in patients with early-stage breast cancer. Treatment consists of dose-dense AC (A: $60 \mathrm{mg} / \mathrm{m}^{2}$ iv.; C: $600 \mathrm{mg} / \mathrm{m}^{2}$ iv.) on day 1 of each 14-day cycle for four cycles, followed by eribulin $1.4 \mathrm{mg} / \mathrm{m}^{2}$ iv. over $2-5 \mathrm{~min}$ on days 1 and 8 every 21 days for four cycles [21]. The primary objective was the ability to complete four cycles of eribulin without a treatment-related dose delay (defined as $>2$ days) or reduction. The study is deemed feasible if 56 or more of the 80 patients can complete the eribulin portion of the study. Exploratory end points include safety, 3-year disease-free survival and overall survival (OS). To date, 55 of 80 patients have been recruited with 46 evaluable for feasibility. The current feasibility rate is $70 \%$ (32 out of 46), with neutropenia being the issue affecting feasibility. However, with administration of growth factor treatment this increased to $85 \%$ (39 out of 46). Given the initial issue with neutropenia the study is being amended to include 
RESEARCH ARTICLE Ramaswami, O'Cathail, Brindley, Silcocks, Mahmoud \& Palmieri

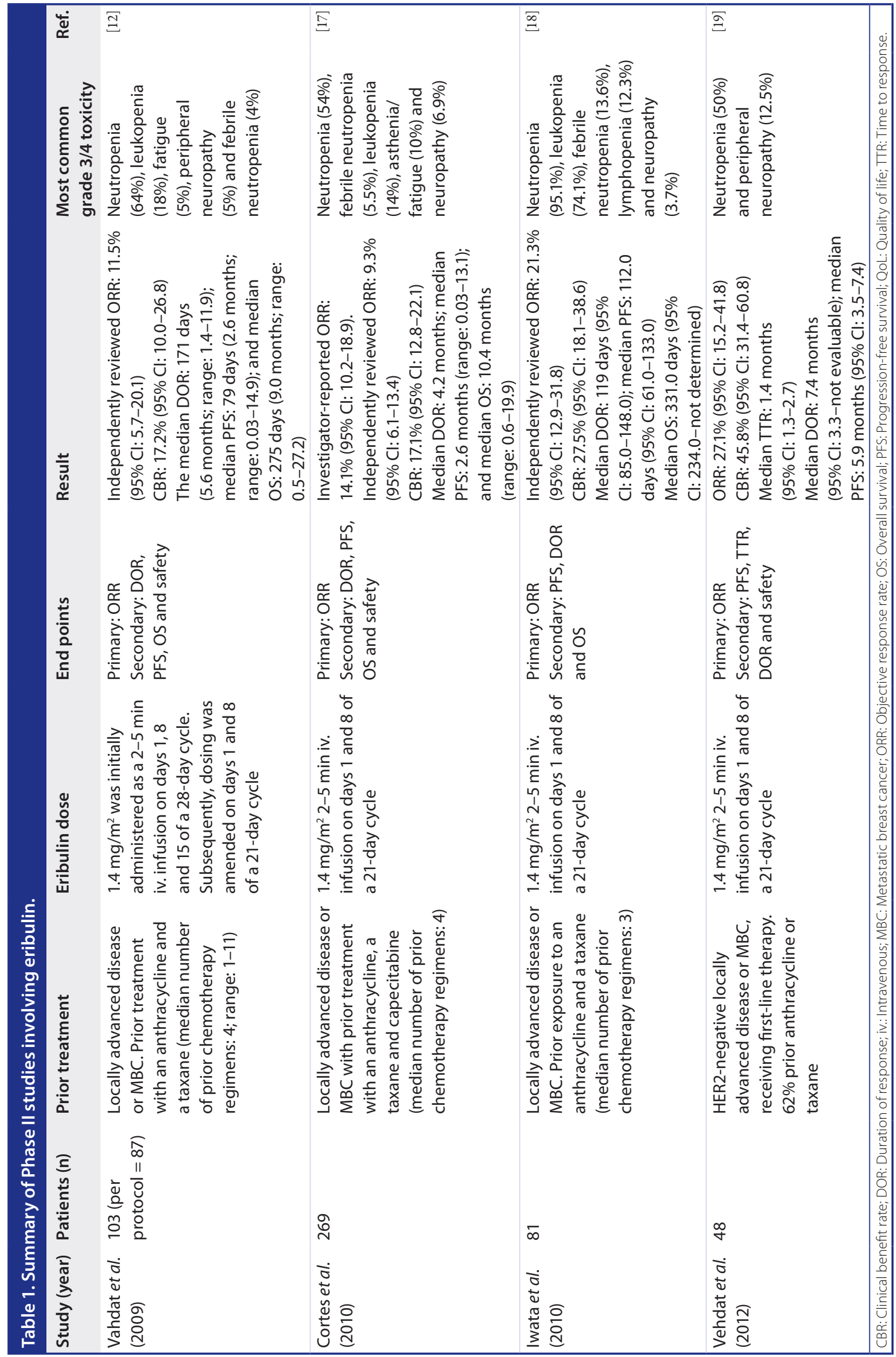




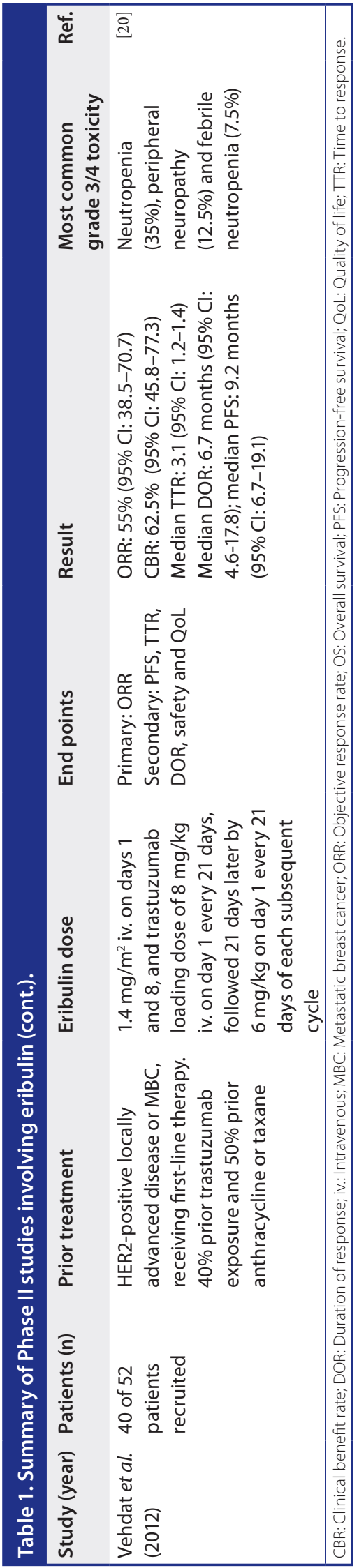

a second cohort that will utilize regimented granulocyte colony-stimulating factor [21].

\section{Phase III studies}

Two Phase III studies have been performed that have tested the efficacy of eribulin in MBC. The first was the EMBRACE study, which was a Phase III open-label study of women with locally recurrent breast cancer or MBC that compared eribulin to a treatment of physician's choice (TPC) [22]. Eribulin was given at $1.4 \mathrm{mg} / \mathrm{m}^{2}$ as a $2-5 \mathrm{~min}$ iv. infusion on days 1 and 8 of a 21-day cycle. In total, 762 women were enrolled and had received between two and five previous chemotherapy regimens, with two or more for advanced disease; these had to include an anthracycline and a taxane, unless contraindicated. The primary end point was OS, and secondary end points included progression-free survival (PFS), objective response rate (ORR) and duration of response. OS was significantly improved in the eribulin group (median: 13.1 months; 95\% CI: 11.8-14.3) compared with the TPC group range (median: 10.6 months; 9.3-12.5; hazard ratio [HR] 0.81; 95\% CI: 0.66-0.99; $\mathrm{p}=0.041)$. Median PFS was 3.7 months (95\% CI: 3.3-3.9) with eribulin and 2.2 months (95\% CI: 2.1-3.4) with TPC (HR: 0.87; 95\% CI: 0.71-1.05; $\mathrm{p}=0.137$ ), while the response rate was $12 \%$ (95\% CI: 9.4-15.5) for eribulin versus 5\% (95\% CI: 2.3-8.4; $\mathrm{p}=0.002)$ for TPC. Significant grade $3 / 4$ toxicities associated with eribulin were asthenia $(9 \%)$, neutropenia (45\%) and peripheral neuropathy (9\%). The most common side effect leading to eribulin discontinuation was peripheral neuropathy (5\% of patients). The mean time to nadir within a cycle was approximately 13 days, and mean time to recovery to grade 2 or lower was approximately 8 days [22].

A second randomized Phase III study of 1102 patients with locally advanced disease or $\mathrm{MBC}$ who have received up to three prior chemotherapy regimens (including an anthracycline and a taxane) compared the efficacy and safety of eribulin $\left(1.4 \mathrm{mg} / \mathrm{m}^{2}\right.$ as a $2-5 \mathrm{~min}$ iv. infusion on days 1 and 8 of a 21-day cycle) versus capecitabine $\left(1250 \mathrm{mg} / \mathrm{m}^{2}\right.$ twice daily on days 1-14 every 21 days) [23]. The coprimary end points of this study were OS and PFS, and secondary end points were: ORR; quality of life; duration of response; 1-, 2- and 3-year survival; and safety. In the study $27 \%$ received study treatment as first-line therapy while 57.4 
Table 2. Clinicopathological characteristics.

\begin{tabular}{|c|c|}
\hline Characteristic & Patients (n) \\
\hline Total patients (n) & 25 \\
\hline Median age; years (range) & $58(28-81)$ \\
\hline $\begin{array}{l}\text { Median time since diagnosis of } \\
\text { MBC to commencing eribulin; } \\
\text { months (range) }\end{array}$ & $32(2-92)$ \\
\hline \multicolumn{2}{|c|}{$\begin{array}{l}\text { Hormone receptor status on diagnosis of } M B C ; \\
n(\%)\end{array}$} \\
\hline $\mathrm{ER}^{+}, \mathrm{PgR}^{+}, \mathrm{HER}_{2}^{+}$ & $4(16)$ \\
\hline $\mathrm{ER}^{+}, \mathrm{PgR}^{-}, \mathrm{HER} 2^{+}$ & $2(8)$ \\
\hline $\mathrm{ER}^{-}, \mathrm{PgR}{ }^{-}, \mathrm{HER}^{+}$ & $2(8)$ \\
\hline $\mathrm{ER}^{+}, \mathrm{PgR}^{+}, \mathrm{HER} 2^{-}$ & $8(32)$ \\
\hline $\mathrm{ER}^{+}, \mathrm{PgR}^{-}, \mathrm{HER}^{-}$ & $4(16)$ \\
\hline $\mathrm{ER}^{-}, \mathrm{PgR}^{-}, \mathrm{HER} 2^{-}$ & $5(20)$ \\
\hline \multicolumn{2}{|l|}{ (Neo)adjuvant chemotherapy; $n$ (\%) } \\
\hline FEC & $3(12)$ \\
\hline FEC-T & $11(44)$ \\
\hline CMF & $1(4)$ \\
\hline EC & $1(4)$ \\
\hline MC & $1(4)$ \\
\hline Trastuzumab & $1(4)$ \\
\hline None/not applicable & $8(32)$ \\
\hline \multicolumn{2}{|l|}{ Adjuvant endocrine therapy } \\
\hline Tamoxifen & $9(36)$ \\
\hline Goserelin + tamoxifen & $2(8)$ \\
\hline Tamoxifen-exemestane & $1(4)$ \\
\hline Anastrozole/letrozole & $4(16)$ \\
\hline $\begin{array}{l}\text { None/not applicable (ER/de novo } \\
\text { metastatic disease) }\end{array}$ & $9(36)$ \\
\hline \multicolumn{2}{|l|}{ De novo metastatic disease; $n$ (\%) } \\
\hline De novo metastatic disease & $5(20)$ \\
\hline \multicolumn{2}{|l|}{ Site of distant metastases; $n$ (\%) } \\
\hline Bone & $13(52)$ \\
\hline Liver & $15(60)$ \\
\hline Lung & $15(60)$ \\
\hline Brain & $4(16)$ \\
\hline Adrenal & $2(8)$ \\
\hline \multicolumn{2}{|c|}{$\begin{array}{l}\text { CMF: Cyclophosphamide, methotrexate and 5-fluorouracil; } \\
\text { EC: Epirubicin and cyclophosphamide; ER: Estrogen receptor; } \\
\text { FEC: 5-fluorouracil, epirubicin and cyclophosphamide; } \\
\text { FEC-T: 5-fluorouracil, epirubicin and cyclophosphamide plus } \\
\text { docetaxel; MBC: Metastatic breast cancer; MC: Non-PEGylated } \\
\text { liposomal doxorubicin and cyclophosphamide; } \\
\text { PgR: Progesterone receptor. }\end{array}$} \\
\hline
\end{tabular}

and $14.7 \%$ received it as second- and third-line treatment, respectively.

Median OS was 15.9 and 14.5 months (HR: 0.879; 95\% CI: $0.770-1.003 ; \mathrm{p}=0.056)$, for eribulin and capecitabine, respectively [23]. OS at 1 year was $64.4 \%$ for eribulin versus $58.8 \%$ for capecitabine $(\mathrm{p}=0.035)$; at 2 years was $32.8 \%$ for eribulin versus $29.8 \%$ for capecitabine $(\mathrm{p}=0.324)$; and at 3 years was $17.8 \%$ for eribulin versus $14.5 \%$ for capecitabine $(\mathrm{p}=0.175)$. PFS (independent review) was 4.1 and 4.2 months (HR: 1.079; 95\% CI: 0.932-1.250; $\mathrm{p}=0.305)$ for eribulin and capecitabine, respectively. ORR (independent review) was 11.0 (95\% CI: 8.5-13.9) and $11.5 \%$ (95\% CI: 8.9-14.5; $\mathrm{p}=0.849$ ), respectively [23]. The median number of treatment cycles was six for eribulin (range: 1-65) and five for capecitabine (0-61), while the duration of treatment was 4.1 months for eribulin (range: $0.7-45.1)$ and 3.9 months for capecitabine (0.7-47). Prespecified subgroup analysis suggested that some subgroups may derive greater benefit with regard to OS with eribulin. These included HER2-negative (15.9 months for eribulin and 13.5 months for capecitabine; HR: 0.838; 95\% CI: 0.715-0.983; $\mathrm{p}=0.030$ ), estrogen receptor-negative (14.4 months for eribulin and 10.5 months for capecitabine; HR: 0.779; 95\% CI: $0.635-0.955)$ and triple-negative (14.4 months for eribulin and 9.4 months for capecitabine; HR: 0.702; 95\% CI: 0.545-0.906) breast cancers.

With regard to adverse effects (all grades) hematological toxicities were much more common with eribulin as compared with capecitabine; neutropenia was seen in 54 versus $16 \%$ and leukopenia in 31.4 versus $10.4 \%$. However, febrile neutropenia was low (2 vs $<1 \%$ ). Nonhematological toxicities were consistent with known data for both agents with capecitabine having a higher rate of hand-foot syndrome ( $0.2 \%$ eribulin vs $45.1 \%$ capecitabine) and diarrhea (14.3 vs $28.8 \%$ ), and eribulin causing more peripheral neuropathy (13 vs $7 \%$ ) and alopecia (35 vs 4\%) [23]. This study establishes that eribulin and capecitabine have similar efficacy in a heterogeneous population of patients with regard to line of treatment and subtype of breast cancer.

Based on the EMBRACE data, eribulin was approved by the US FDA in November 2010, and was subsequently approved in Europe in March 2011 [24] for the treatment of patients with locally advanced or recurrent breast cancer that had progressed after two lines of chemotherapy; prior therapy has to include an anthracycline and taxane unless deemed unsuitable for these patients. Within England all new cancer agents are considered by the National Institute for Health and Care Excellence (NICE) and in Scotland by the Scottish Medicine Consortium 
for funding approval prior to use within the licensed indication. NICE considered that the incremental cost-effectiveness ratios for eribulin versus TPC within the EMBRACE population was at least $\mathrm{GB} £ 68,000$ per quality-adjusted life year gained and, therefore, did not recommend its funding [25]. The Scottish Medicine Consortium calculated that compared with TPC the cost per quality-adjusted life year was $G B £ 81,852$ for eribulin [26] and, therefore, again did not recommended its funding and use. The issue of accessing and funding high-cost drugs led to the establishment of the Cancer Drugs Fund (CDF) for drugs not funded by NICE in October 2010 [27]. The CDF applies solely to England. Drugs and how they were accessed was initially determined regionally via a clinically led panel, but in April 2013 a national CDF list was established [28]. Eribulin has been available in London via the CDF since September 2011 [29]. To be eligible patients have to have locally advanced breast cancer or MBC that has progressed after at least two chemotherapeutic regimens for advanced disease. Prior therapy should have included an anthracycline and a taxane unless patients were not suitable for these treatments.

\section{Materials \& methods}

All requests for eribulin to the London CDF between the 29 September 2011 and 28 February 2013 for patients with locally advanced breast cancer or MBC attending Imperial College Healthcare NHS Trust (London, UK) were reviewed. Patients commencing on treatment were identified and their case notes and radiology reviewed. To be eligible for eribulin via the CDF patients had to meet the criteria previously described above. The histopathological and (neo)adjuvant treatment details, site of disease when commencing eribulin, prior metastatic treatment including rechallenging with anthracyclines or taxanes, number of cycles of eribulin, time-to-progression (TTP), best response and outcome, as well as any treatment given after completing eribulin, were recorded. All patients commenced on eribulin $1.23 \mathrm{mg} / \mathrm{m}^{2}$ (equivalent to $1.4 \mathrm{mg} / \mathrm{m}^{2}$ eribulin mesylate) iv. on days 1 and 8 of every 21-day cycle, unless otherwise stated.

TTP was defined as time from first eribulin treatment to date of progression (including progression-related death) with death from other causes and withdrawal as censoring events. OS was defined as time from first eribulin treatment to death from any cause with censoring events, such as alive on last follow-up examination. Follow-up was administratively censored on

Table 3. Breast cancer treatment received in

metastatic setting prior to and following

\section{eribulin.}

Treatment

Patients ( $\mathrm{n}$ )

Endocrine therapy

Tamoxifen 2

Al alone 18

Al + FEC chemotherapy 2

$\mathrm{Al}+$ trastuzumab

Fulvestrant 8

Steroid sulfatase inhibitor

Goserelin alone 1

Goserelin + Al 1

Goserelin + Al + trastuzumab 1

Median lines of prior endocrine $\quad 1(0-5)$

therapy for MBC (range)

Chemotherapy

Capecitabine alone 20

Capecitabine/lapatinib 4

Capecitabine/trastuzumab 1

Paclitaxel 16

Docetaxel 3

Docetaxel/carboplatin

FEC

FEC-T

MC 4

Vinorelbine 3

Gemcitabine/carboplatin

CMF

Paclitaxel/trastuzumab

Docetaxel/trastuzumab 2

FEC/trastuzumab 1

Median lines of prior chemotherapy 3 (1-4)

for MBC (range)

Median lines of prior chemotherapy $3(2-5)$

(Adj and MBC) (range)

Treatment for metastatic breast cancer after eribulin $(n=11)$

\begin{tabular}{ll} 
Vinorelbine & 6 \\
Epirubicin & 1 \\
Nab-paclitaxel & 1 \\
Paclitaxel & 1 \\
MC & 1 \\
Gemcitabine/carboplatin & 1 \\
\hline Adj: Adjuvant; Al: Aromatase inhibitors; \\
CMF: Cyclophosphamide, methotrexate and fluorouracil; \\
FEC: 5-fluorouracil, epirubicin and cyclophosphamide; \\
FEC-T: 5-fluorouracil, epirubicin and cyclophosphamide plus \\
docetaxel; MBC: Metastatic breast cancer; MC: Non-PEGylated \\
liposomal doxorubicin and cyclophosphamide.
\end{tabular}


Table 4. Details of eribulin treatment, response and toxicity.

\begin{tabular}{ll} 
Characteristic & Patients (n) \\
Median number of cycles of eribulin, & $4(1-15)$ \\
$\mathrm{n}$ (range) & \\
Response; $\boldsymbol{n}$ (\%) & \\
Complete response & $0(0)$ \\
Partial response & $4(16)$ \\
\hline Stable disease & $8(32)$ \\
\hline Progressive disease & $10(40)$ \\
Clinical benefit rate & $9(41)$ \\
Not assessable & $3(12)$ \\
Toxicity; $\boldsymbol{n}$ (\%) & \\
None & $9(36)$ \\
Neuropathy; $n$ (\%) & \\
Grade 1 & $3(12)$ \\
Grade 2 & $1(4)$ \\
Grade 3 & $1(4)$ \\
Neutropenia; $n$ (\%) & \\
Grade 1 & $5(20)$ \\
Grade 2 & $3(12)$ \\
Other & \\
Nausea/vomiting (grade 1) & $3(12)$ \\
Diarrhea (grade 1) & $1(4)$ \\
Fatigue (grade 2) & $2(8)$ \\
Abnormal liver function tests & $1(4)$ \\
\hline $\begin{array}{l}\text { Clinical benefit rate included complete or partial response, or } \\
\text { stable disease of at least 6-month duration. }\end{array}$ \\
\hline
\end{tabular}

28 February 2013. Survival analyses used the Kaplan-Meier method and Cox's proportional hazards model. Exact confidence limits were calculated for binomial proportions, with calculations performed in Statav 12.1 (StataCorp, TX, USA).

Response evaluation criteria in solid tumors was used to define response [30] and adverse events and laboratory abnormalities were graded according to the National Cancer Institute Common Terminology Criteria for Adverse Events, version 3.0 [31]. ORR was defined as the rate of complete response or partial response; while clinical benefit rate (CBR) was defined as the rate of complete plus partial response plus stable disease of at least 6 months' duration.

\section{Results}

Twenty-five patients were treated via the London CDF with eribulin between September 2011 and February 2013. Baseline characteristics of patients and treatments prior to and following eribulin therapy are summarized in Tables $2 \& 3$, respectively.
In those assessable, the ORR was $18 \%$ (four out of 22; 95\% CI: 5.2-40.3\%). Three patients did not undergo midtreatment assessment: one died suddenly after the first cycle, the cause of which is unknown, and another two patients discontinued secondary to toxicity prior to assessment (Table 4). The CBR was $41 \%$ (nine out of 22; 95\% CI: 21.0-64.0), the overall median TTP was 4.08 months (95\% CI: 2.40-8.95) and the median OS was 5.89 months (95\% CI: $3.88-8.75$ ) (Figure 1). Five patients remain on treatment at time of analysis, with 55\% (11 out of 20) having gone on to receive further chemotherapy at disease progression (Table 3).

In total, $44 \%$ (11 out of 25 ) of patients had been rechallenged with an anthracycline or taxane during their disease course prior to eribulin. In those assessable, when the response to treatment was compared based on rechallenge there was a numerical but nonsignificant difference in ORR favoring those not rechallenged of 28.5 (four out of 14) versus $0 \%$ (zero out of eight), respectively $(\mathrm{p}=0.254)$, but there was a significant difference in CBR of 64 (nine out of 14) versus $0 \%$ (zero out of eight; $p=0.0055$ ). TTP was significantly longer in those not rechallenged; median TTP was 8.95 versus 2.60 months (HR: 9.18; 95\% CI: 2.26-37.38; $\mathrm{p}=0.002$ ); while OS was numerically longer in those rechallenged, the difference was not statistically significant: median OS was 6.94 versus 3.82 months (HR: $1.16 ; 95 \%$ CI: 0.44-3.05; $\mathrm{p}=0.770$ ) (Figure 2).

Of note, one patient received eribulin concurrently with trastuzumab with no problems. In one case eribulin was given at $0.7 \mathrm{mg} / \mathrm{m}^{2}$ due to severely deranged liver function tests due to metastatic disease. However, a grade 3 asymmetrical, sensorimotor polyneuropathy developed that led to cessation of eribulin; this subsequently improved. Despite discontinuing after 1.5 cycles there was clear evidence of clinical and biochemical improvement, as evidenced by improving liver function tests and a fall in CA153 from 687 to 397 kunits/l. A scan subsequently performed for clinical reasons showed evidence consistent with stable disease. Neutropenia and neurotoxicity were the most common toxicities, and the majority were grade 1 and 2 (Table 4).

\section{Discussion}

Chemotherapy is a key treatment in the management of endocrine refractory estrogen receptorpositive breast cancer as well as the treatment of choice in triple-negative breast cancer. In 
HER2-positive breast cancer chemotherapy is a key backbone with which HER2-based therapy is combined either as separate agents given concurrently [32] or, more recently, the use of chemotherapy conjugated to a HER2 antibody [33]. The potential chemotherapeutic options for patients requiring chemotherapy for $\mathrm{MBC}$ include anthracycline-based regimens [34]; taxane-based therapy, either as a single agent or combined with capecitabine [35] or bevacizumab [36,37]; singleagent capecitabine [38] or capecitabine combined with ixabepilone [39] or bevacizumab [40]; platinum-based treatments [41]; vinorelbine with or without gemcitabine [42,43]; and a cyclophosphamide, methotrexate and 5-fluorouracil combination [44]. For those with prior anthracycline and taxane exposure, rechallenging is also an option [45]; and in HER2-positive disease trastuzumab [32], lapatinib [46], pertuzumab [47] and T-DM1 [33] are also options. Eribulin, therefore, joins a large list of potential therapeutic options; based on current data it is a treatment option for patients who have been previously exposed to an anthracycline, a taxane and capecitabine [22], and recent data in a more heterogeneous group have shown it is an alternative treatment option to capecitabine [23].

In this unselected cohort of patients all but two had prior treatment with an anthracycline, a taxane and capecitabine. One case was not treated with an anthracycline due to prior exposure during treatment for non-Hodgkin's lymphoma, and one patient had not received capecitabine prior to eribulin. In those patients who were assessable the ORR was $18 \%$ (four out of 22), while the TTP for the whole cohort was 4.1 months. While it is clearly difficult to compare across this study and a randomized study [22], the ORR and TTP are comparable to the data within EMBRACE. However, the OS is shorter in our patient group: 5.9 versus 13.1 months within EMBRACE. The difference in OS may be explained by the prior treatment received before the eribulin, with $96 \%$ (24 out of 25) in the current study having been exposed to capecitabine prior to eribulin as compared with $74 \%$ in EMBRACE. Therefore, the potential to use an agent with known efficacy was much more limited in our patients following progression. However, the similar ORR and TTP between the current cohort and those within EMBRACE suggests that eribulin is still effective in a patient population that has been exposed to capecitabine. Furthermore, $44 \%$ of cases in the current cohort had been rechallenged with an anthracycline or a taxane and it is not clear from the EMBRACE study what proportion of those that received eribulin did so following rechallenging with these agents. The presence within this current cohort of a high proportion of such patients could have impacted the OS data. Within EMBRACE, $50 \%$ of patients randomized to eribulin went onto receive further chemotherapy postprogression, as compared with $44 \%$ of cases within this cohort. The difference seen in OS may be a reflection of this, as well as the relative efficacy of postprogression treatment in the two cohorts.

In those progressing on eribulin, 55\% (11 out of 20) received further chemotherapy, the majority receiving the alternative antimicrotubule agent vinorelbine. The degree of crossresistance between vinorelbine and eribulin is unknown, as is the efficacy. Four of the remaining five were rechallenged
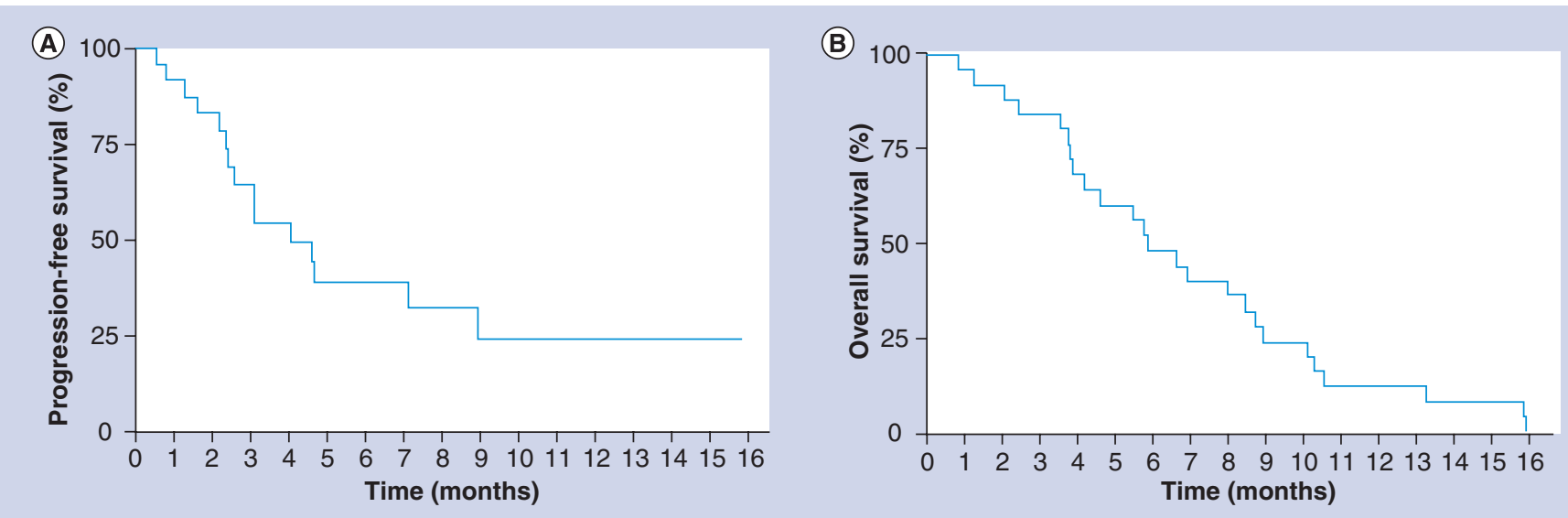

Figure 1. Progression-free survival and overall survival for the whole of the treated cohort. (A) Progression-free survival and (B) overall survival. 

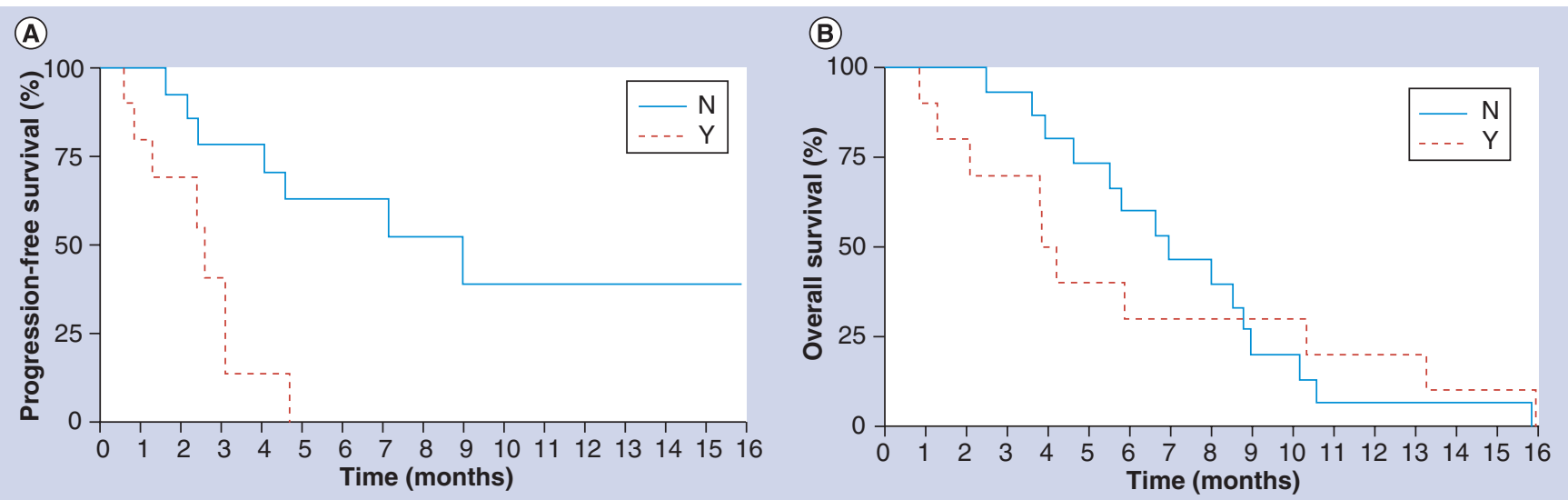

Figure 2. Progression-free survival and overall survival for patients rechallenged with anthracycline and/or taxane versus not rechallenged. (A) Progression-free survival and (B) overall survival for $\mathrm{Y}$ versus $\mathrm{N}$.

$\mathrm{N}$ : Not rechallenged; Y: Rechallenged with anthracycline and/or taxane.

with an anthracycline or taxane; again, potential efficacy of such a treatment is unclear [45]. In total, $50 \%$ of eribulin-treated patients within EMBRACE received further chemotherapy postprogression, although the treatment regimens used in these cases are not available [22]. Such data, as well as the efficacy of this postprogression treatment, would clearly be of value to support and guide systemic treatment decisions beyond eribulin. With regard to the difference seen in OS between the EMBRACE population and this cohort it may be a reflection of the proportion who received subsequent treatment as well as the relative efficacy of this postprogression treatment.

In total, 44\% (11 out of 25) of the cohort had been re-exposed to either an anthracycline or a taxane during their adjuvant and/or metastatic treatment, and all had received capecitabine. There was a significant difference in CBR and TTP compared with those not rechallenged: while OS was numerically longer it was not statistically significant. The reasons for these differences may be due to the number of lines of prior treatment, which was higher in the rechallenged group as compared with those not rechallenged, with a median of four and three cycles of prior chemotherapy, respectively (Table 5). In addition, the reutilization of key classes of chemotherapy agents could be indicative of disease that is relatively refractory or that has exhausted reasonable treatment options. Furthermore, the potential effects on re-exposing a tumor to the same class of agents on subsequent treatments is unclear: this may be particularly important in those rechallenged with a taxane given the mechanism of action of eribulin. This observation is hypothesis generating given the small numbers and retrospective nature of the study. However, the benefit of eribulin based on rechallenging should be explored with the EMBRACE data set [22]. Such data are important as it may identify patients who are less likely to derive benefit from eribulin; this is particularly important in MBC given the incurable nature of the disease and the importance of quality of life.

Eribulin was well tolerated in this cohort of heavily pretreated patients with the majority of toxicities being grade 1 and 2, and, as expected, neutropenia and neurotoxicity were the most common. The case of grade 3 neurotoxicity was in a patient with severely deranged liver function tests who was treated with the recommended dose of $0.7 \mathrm{mg} / \mathrm{m}^{2}$. It is known that liver dysfunction can significantly increase the exposure to eribulin by 1.75 - to 2.48 -fold [14], and that caution is, therefore, required in such cases.

The current dose modifications for eribulin where there is liver dysfunction is based on the Child-Pugh score [16]. However, the Child-Pugh score was developed to assess the operative risk in patients requiring surgical portosystemic shunts due to chronic liver disease [48], with the score representing the collective knowledge of surgeons accumulated over time performing this procedure. It was subsequently modified by Pugh and colleagues to describe the outcome of patients undergoing surgical ligation of esophageal varices due to chronic liver disease [49]. Hence, the Child-Pugh Score was developed for a specific prognostic purpose, and was not developed for or intended to be used in the dosing of drugs that may be metabolized or excreted via the liver. 
Ordinarily, within oncology, both trial entry and dose modifications for such drugs have been based on the degree of derangement of the liver function tests (namely transaminases and alkaline phosphatase) from the upper limit of normal [17]. Given the data that even at the lower dose using the Child-Pugh score there is a significant effect on eribulin exposure [50], and given the fact that the Child-Pugh score was not developed for this purpose, consideration should be given to further pharmacokinetics studies that are based on liver function as measured by transaminases and alkaline phosphatase. This would be helpful in clarifying if a lower dose or modified treatment regimen could reduce the eribulin exposure and, therefore, reduce the risks of eribulin accumulation and, therefore, associated toxicity in such cases. Furthermore this would be helpful in developing more relevant dose modification criteria. This is particularly important given the palliative nature of the treatment and the need to avoid unnecessary toxicity with its potential affects on quality of life, as well as ensuring that the drug is not unnecessarily discontinued.

One sudden unexplained death after the first treatment was observed, and its link to eribulin is unknown. There are no previous reports in the literature of sudden deaths with eribulin and it is not possible to establish any causal link between the death and eribulin treatment. In an open-label ECG study of 26 patients eribulin was found to cause QT prolongation on day 8, independent of eribulin concentration, with no QT prolongation observed on day 1 [50]. Therefore, ECG monitoring is recommended if therapy is initiated in patients with bradyarrhythmia, electrolyte abnormalities, or concomitantly to drugs that prolong the QT interval, as well as avoidance of use in congenital long-QT syndrome. To the best of our knowledge this patient had no prior cardiac history, nor were they receiving any concomitant medications.

In conclusion, in the setting of a routine clinical practice involving heavily pretreated patients eribulin has evidence of clinical activity and is well tolerated. Of interest, a comparison of patients based on a history of rechallenging either with anthracyclines or taxanes appears to suggest that these patients with no such history may derive greater benefit from eribulin. This requires confirmation in a larger data set.

\section{Conclusion \& future perspective}

Eribulin is now established as a therapeutic option in women who have been previously exposed to an anthracycline and taxane [22] and, in a heterogeneous population, has recently been shown to be equivalent to capecitabine [23]. Studies are currently investigating its efficacy as a first-line agent in both HER2-negative and -positive disease. Furthermore, its feasibility in a potential adjuvant regimen is being explored. Taxanes are currently the most widely used antimicrotubule agents in the treatment of breast cancer. However, paclitaxel and docetaxel, formulated in Cremophor ${ }^{\circledR}$ EL (BASF Corp., Ludwigshafen, Germany) and polysorbate 80, respectively, are diluents associated with hypersensitivity reaction that necessitate premedication with steroids. Furthermore, paclitaxel and docetaxel require polyethylene-lined rather than PVC sets given the associated leeching of plasticizer di-(2-ethylhexyl) phthalate that occurs with PVC infusion sets. Given the water soluble nature of eribulin it does not require premedication or particular infusion sets and is not associated with infusion reactions, it also has a shorter infusion time. Therefore, eribulin represents an alternative nontaxane antimicrotubular agent; however, formal studies are required to assess its relative efficacy to these agents. Recent subgroup analysis from the comparative study of eribulin versus capecitabine suggests certain groups may derive particular benefit from eribulin [23]; this again requires formal testing in the context of clinical studies. With regard to subsequent treatments following eribulin, information is needed regarding the response and toxicities to such treatments. This is important to guide subsequent decision-making and is particularly relevant to other antimicrotubule agents such as vinorelbine and ixabepilone. To date, Phase III data with eribulin have been in the context of a single agent $[22,23]$ and one Phase II study has explored it in sequence with AC chemotherapy [21]. However, its efficacy may be improved by combining it with other chemotherapy agents, such as HER2-directed or antiangiogenesis agents. Such combination treatments, particularly with chemotherapy, will probably require modification to the dose and/or regimen.

Table 5. Median lines of chemotherapy in patients rechallenged versus not rechallenged with anthracycline or taxane in the metastatic setting.

\begin{tabular}{lll} 
Median lines of chemotherapy & \multicolumn{2}{l|}{ Rechallenged with anthracycline or taxane } \\
\cline { 2 - 3 } & Yes $(\boldsymbol{n}=11)$ & No $(\boldsymbol{n}=14)$ \\
Adjuvant and metastatic (range) & $4(3-5)$ & $3(2-4)$ \\
Metastatic only (range) & $3(2-4)$ & $3(1-3)$ \\
\hline
\end{tabular}




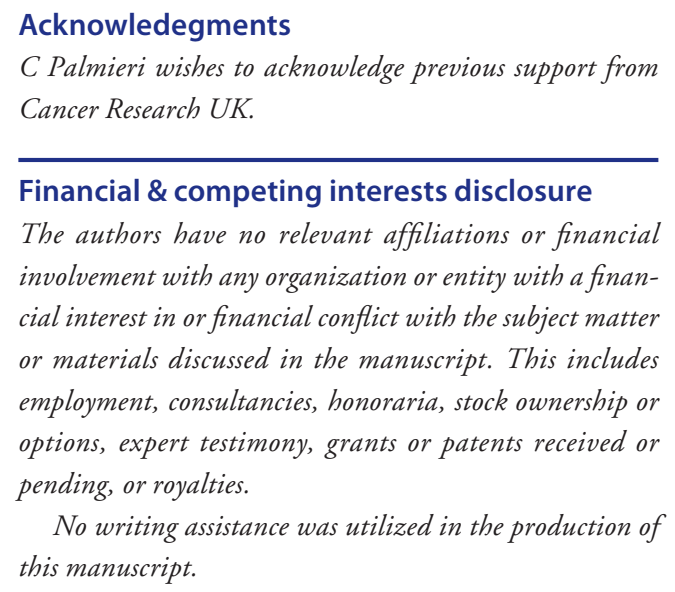

No writing assistance was utilized in the production of this manuscript.

Ethical conduct of research

The authors state that they have obtained appropriate institutional review board approval or have followed the principles outlined in the Declaration of Helsinki for all human or animal experimental investigations. In addition, for investigations involving human subjects, informed consent has been obtained from the participants involved.

\section{Open access}

This work is licensed under the Creative Commons Attribution-NonCommercial 3.0 Unported License. To view a copy of this license, visit http://creativecommons.org/ licenses/by-nc-nd/3.0/

\section{EXECUTIVE SUMMARY}

\section{Background}

- Eribulin mesylate is a synthetic analog of halichondrin B, a naturally occurring product isolated from Halichondria okadai.

- Eribulin is an inhibitor of microtubule dynamics.

- Hepatic impairment decreases the clearance of eribulin.

- The EMBRACE study showed that eribulin as compared with treatment of physician's choice resulted in a significant increase in overall survival in heavily pretreated locally advanced disease/metastatic breast cancer.

- Eribulin is equivalent to capecitabine in locally advanced disease/metastatic breast cancer that has been exposed to up to three prior chemotherapy regimens.

- The most common toxicities are neutropenia, fatigue, alopecia and peripheral neuropathy.

- It has not been deemed to be a cost-effective treatment in the UK.

- In England eribulin can be accessed via the Cancer Drugs Fund.

\section{Results}

- In 25 women receiving treatment via the Cancer Drugs Fund the objective response rate was $18 \%$ and the clinical benefit rate was $55 \%$.

- The median time to progression was 4.08 months and overall survival was 5.89 months.

- Clinical benefit rate and time to progression were significantly longer in those patients who had not been rechallenged with an anthracycline or taxane.

- Treatment was well tolerated.

- One case of neurotoxicity on the background of deranged liver function tests (LFTs) was recorded despite a dose reduction to the lowest dose. This resolved on discontinuation of the drug.

- One sudden unexplained death was recorded.

\section{Conclusion}

- The use of eribulin in a routine clinical practice in a heavily pretreated population does result in clinical benefit and overall is well tolerated.

- Caution is required in patients with deranged LFTs even when using the recommend $0.7 \mathrm{mg} / \mathrm{m}^{2}$ dose. Studies of alternative doses or regimens of eribulin are required in patients with severely deranged LFTs.

- A comparison of patients based on a history of rechallenging either to anthracyclines or taxanes appears to suggest that these patients with no such history may derive greater benefit from eribulin. This requires confirmation in a larger data set. 


\section{References}

Papers of special note have been highlighted as:

- of interest

-. of considerable interest

1 Hirata Y, Uemura D.

Halichondrins - antitumor polyether macrolides from a marine sponge. Pure Appl. Chem. 58, 701-710, (1986).

-. Describes the purification of halichondrins, norhalichondrins and homohalichondrins from Halichondria okadai, and the structure and initial in vitro activity on melanoma and leukemia cell lines.

2 Tachibana K, Scheuer PJ, Tsukitani Y et al. Okadaic acid, a cytotoxic polyether from two marine sponges of the genus Halichondria. J. Am. Chem. Soc. 103, 2469 (1981).

3 Aicher TD, Buszek KR, Fang FG et al. Total synthesis of halichondrin $\mathrm{B}$ and norhalichondrin B. J. Am. Chem. Soc. 114, 3162-3164 (1992).

-. Describes the synthesis of halichondrin B.

4 Wang Y, Habgood G, Christ W, Kishi Y, Littlefield B, Yu M. Structure-activity relationships of halichondrin $\mathrm{B}$ analogues modifications at C30-C38. Bioorg. Med. Chem. Lett. 10, 1029-1032 (2000).

5 Littlefield BA, Palme MH, Seletsky BM et al. Macrocyclic Analogs and Methods of Their Use and Preparation. US Patent Application 09/334,488. US Patent Office, DC, USA (1999).

6 Towle MJ, Salvato KA, Budrow J et al. In vitro and in vivo anticancer activities of synthetic macrocyclic ketone analogues of halichondrin B. Cancer Res. 61, 1013-1021 (2001).

-. In vitro and in vivo activity of ER-086526, which was taken forward for clinical development and subsequently named eribulin.

7 Kuznetsov G, TenDyke K, Yu M et al. Antiproliferative effects of halichondrin B analog eribulin mesylate (E7389) against paclitaxel-resistant human cancer cells in vitro. Proc. Am. Assoc. Cancer Res. Abstract C58 (2007).

8 Jordan MA, Kamath K, Manna T et al. The primary antimitotic mechanism of action of the synthetic halichondrin E7389 is suppression of microtubule growth. Mol. Cancer Ther. 4, 1086-1095 (2005).

9 Synold TW, Morgan RJ, Newman EM et al. A Phase I pharmacokinetic, target validation study of the novel anti-tubulin agent E7389: a California Cancer consortium trial. J. Clin. Oncol. 23, Abstract 3036 (2005).
10 Goel S, Mita AC, Mita M et al. A Phase I study of eribulin mesylate (E7389), a mechanistically novel inhibitor of microtubule dynamics, in patients with advanced solid tumors. Clin. Cancer Res. 15, 4207-4212 (2009).

11 Tan AR, Rubin EH, Walton DC et al. Phase I study of eribulin mesylate (E7389) administered once every 21 days in patients with advanced solid tumors. Clin. Cancer Res. 15, 4213-4218 (2009).

12 Vahdat LT, Pruitt B, Fabian CJ et al. Phase II study of eribulin mesylate, a halichondrin B analog, in patients with metastatic breast cancer previously treated with an anthracycline and a taxane. J. Clin. Oncol. 27, 2954-2961 (2009).

- Phase II study in locally recurrent/metastatic breast cancer that initially utilized eribulin

$1.4 \mathrm{mg} / \mathrm{m}^{2}$ intravenously on days 1,8 and 15 of a 28-day cycle. Neutropenia at day 15 resulted in alternative regimen of eribulin on days 1 and 8 of a 21-day cycle being administered. Provides evidence of the activity of eribulin in recurrent breast cancer.

13 Spira AI, Iannotti NO, Savin MA et al. Phase II study of eribulin mesylate (E7389), a mechanistically novel inhibitor of microtubule dynamics, in patients with advanced non-small cell lung cancer (NSCLC). J. Clin. Oncol. 25(Suppl.18), Abstract 7546 (2007).

-. Phase II study in lung cancer that demonstrates that $1.4 \mathrm{mg} / \mathrm{m}^{2}$ intravenous eribulin mesylate on days 1,8 and 15 of a 28-day cycle was not feasible and resulted in the alternative regimen of eribulin on days 1 and 8 of a 21-day cycle being administered.

14 Mukohara T, Nagai S, Mukai H et al. Eribulin mesylate in patients with refractory cancers: a Phase I study. Invest. New Drugs 30, 1926-1933 (2012).

- A Phase I study documenting pharmacokinetics and the maximum tolerated dose for the modified eribulin regimen of treatment on days 1 and 8 of a 21-day cycle. Establishes eribulin $1.4 \mathrm{mg} / \mathrm{m}^{2}$ administered on days 1 and 8 of a 21-day cycle as being the best tolerated, and was taken forward in all subsequent studies.

15 Dubbelman AC, Rosing H, Jansen RS et al. Mass balance study of $\left[{ }^{14} \mathrm{C}\right]$ eribulin in patients with advanced solid tumors. Drug Metab. Dispos. 40, 313-321 (2012).

-. Determines the metabolism and excretion of eribulin after a single dose of ${ }^{14} \mathrm{C}$-eribulin in patients with advanced solid tumors. Demonstrates that the majority of eribulin is excreted unchanged in feces.
16 Devriese LA, Witteveen PO, Marchetti S et al. Pharmacokinetics of eribulin mesylate in patients with solid tumors and hepatic impairment. Cancer Chemother. Pharmacol. 70, 823-832 (2012).

-. Documents the pharmacokinetics of eribulin in Child-Pugh A and B liver dysfunction and need for dose modifications in such cases.

17 Cortes J, Vahdat L, Blum JL et al. Phase II study of the halichondrin B analog eribulin mesylate in patients with locally advanced or metastatic breast cancer previously treated with an anthracycline, a taxane, and capecitabine. J. Clin. Oncol. 28, 3922-3928 (2010).

-. Phase II study demonstrating the efficacy of eribulin in locally advanced or metastatic breast cancer previously treated with an anthracycline, a taxane and capecitabine.

18 Iwata $\mathrm{H}$, Aogi $\mathrm{K}$, Masuda $\mathrm{N}$ et al. Efficacy and safety of eribulin in Japanese patients (pts) with advanced breast cancer. J. Clin. Oncol. 28(Suppl. 15), Abstract 1081 (2010).

19 Vahdat L, Schwartzberg L, Glück S et al. Results of a Phase 2, multicenter, single-arm study of eribulin mesylate as first-line therapy for locally recurrent or metastatic HER2-negative breast cancer. Cancer Res. 72(Suppl. 24), Abstract P1-12-02 (2012).

- Initial Phase II data of single-agent eribulin as first-line therapy in HER2-negative locally advanced/metastatic breast cancer.

20 Vahdat L, Schwartzberg L, Wilks S et al. Eribulin mesylate + trastuzumab as first-line therapy for locally recurrent or metastatic HER2-positive breast cancer: results from a Phase 2, multicenter, single-arm study. Cancer Res. 72 (24 Suppl.), Abstract P5-20-04 (2012).

- Initial Phase II data of combining eribulin with trastuzumab as first-line therapy in HER2-positive locally advanced/metastatic breast cancer.

21 Traina TA, Hudis C, Fornier M et al. Adjuvant treatment of early-stage breast cancer with eribulin mesylate following dose-dense doxorubicin and cyclophosphamide: preliminary results from a Phase 2, single-arm feasibility study. Cancer Res. 72 (24 Suppl.), Abstract P1-13-11 (2012).

- Assessment of the feasibility of administering eribulin following dose-dense doxorubicin plus cyclophosphamide.

22 Cortes J, O'Shaughnessy J, Loesch D et al; EMBRACE (Eisai Metastatic Breast Cancer Study Assessing Physician's Choice Versus E7389) investigators. Eribulin monotherapy 
versus treatment of physician's choice in patients with metastatic breast cancer (EMBRACE): a Phase 3 open-label randomised study. Lancet 377, 914-923 (2011).

-. Pivotal Phase III study of eribulin versus physician's choice, which demonstrates improvement in overall survival favoring eribulin. Formed the basis of its subsequent US and European license.

23 Kaufman PA, Awada A, Twelves C et al. A Phase III, open-label, randomized, multicenter study of eribulin mesylate versus capecitabine in patients with locally advanced or metastatic breast cancer previously treated with anthracyclines and taxanes. Cancer Res. 72, Abstract S6-6 (2012).

-. Compares eribulin and capecitabine in metastatic breast cancer. Second Phase III trial in breast cancer.

24 European Medicines Agency. Assessment Report for Halaven Eribulin Procedure No. EMEA/H/C/002084 Committee for Medicinal Products for Human Use (CHMP). European Medicines Agency, London, UK (2011).

25 National Institute for Health and Care Excellence. Final Appraisal Determination Eribulin for the Treatment of Locally Advanced or Metastatic Breast Cancer. National Institute for Health and Care Excellence, London, UK (2011).

26 Eribulin $0.44 \mathrm{mg} / \mathrm{ml}$ Solution for Injection (Halaven $^{\circledR}$ ) SMC No. (726/11). Scottish Medicine Consortium, Glasgow, UK (2011).

27 Interim Cancer Drug Funding, Gateway reference: 14617. Department of Health, London, UK (2010).

28 Standard Operating Procedures: The Cancer Drugs Fund (CDF). Guidance to Support Operation of the CDF in 2013-2014. NHS Commissioning Board, Redditch, UK (2013).

29 NHS London Strategic Health Authority. Report on Interim Cancer Drugs Funding Arrangement for London (1 October 2010 - 31 March 2011) London Cancer New Drugs Group, London, UK (2011).

30 Therasse P, Arbuck SG, Eisenhauer EA et al. New guidelines to evaluate the response to treatment in solid tumors. European
Organization for Research and Treatment of Cancer, National Cancer Institute of the United States, National Cancer Institute of Canada. J. Natl Cancer Inst. 92, 205-216 (2000).

31 National Cancer Institute. National Cancer Institute Common Terminology Criteria for Adverse Events, version 3.0. National Cancer Institute, MD, USA (2006).

32 Slamon DJ, Leyland-Jones B, Shak S et al. Use of chemotherapy plus a monoclonal antibody against HER2 for metastatic breast cancer that overexpress HER2. N. Engl. J. Med. 344, 783-792 (2001).

33 Krop IE, Beeram M, Modi S et al. Phase I study of trastuzumab-DM1, an HER2 antibody- drug conjugate, given every 3 weeks to patients with HER2-positive metastatic breast cancer. J. Clin. Oncol. 28, 2698-2704 (2010).

34 French Epirubicin Study Group. A prospective randomized Phase III trial comparing combination chemotherapy with cyclophosphamide, fluorouracil and either doxorubicin or epirubicin. J. Clin. Oncol. 6, 679-688 (1988).

35 O'Shaughnessy J, Miles D, Vukelja S et al. Superior survival with capecitabine plus docetaxel combination therapy in anthracycline-pretreated patients with advanced breast cancer: Phase III trial results. J. Clin. Oncol. 20, 2812-2823 (2002).

36 Miller K, Wang M, Gralow J et al. Paclitaxel plus bevacizumab versus paclitaxel alone for metastatic breast cancer. N. Engl. J. Med. 357 , 2666-2676 (2007).

37 Miles DW, Chan A, Dirix LY et al. Phase III study of bevacizumab with docetaxel or docetaxel with placebo for the first line treatment of human epidermal growth factor receptor- 2 negative metastatic breast cancer (mBC). J. Clin. Oncol. 28, 3239-3247 (2010).

38 Seidman AD, O’Shaughnessy J, Misset JL. Single-agent capecitabine: a reference treatment for taxane-pretreated metastatic breast cancer? Oncologist 7(Suppl. 6), 20-28 (2002).

39 Thomas ES, Gomez HL, Li RK et al. Ixabepilone plus capecitabine for metastatic breast cancer progressing after anthracycline and taxane treatment. J. Clin. Oncol. 25, 5210-5217 (2007).

40 Miller KD, Chap LI, Holmes FA et al. Randomized Phase III trial of capecitabine compared with bevacizumab plus capecitabine in patients with previously treated metastatic breast cancer. J. Clin. Oncol. 23, 792-799 (2005).

41 Carrick S, Ghersi D, Wilcken N, Simes J. Platinum containing regimens for metastaticbreast cancer. Cochrane Database Syst. Rev. 3, CD003374 (2004).

42 Zelek L, Barthier S, Riofrio M et al. Weekly vinorelbine is an effective palliative regimen after failure with anthracyclines and taxanes in metastatic breast carcinoma. Cancer 92, 2267-2272 (2001).

43 Martín M, Ruiz A, Muñoz M et al. Gemcitabine plus vinorelbine versus vinorelbine monotherapy in patients with metastatic breast cancer previously treated with anthracyclines and taxanes: final results of the Phase III Spanish Breast Cancer Research Group(GEICAM) trial. Lancet Oncol. 8, 219-225(2007).

44 O’Byrne KJ, Koukourakis MI, Saunders MP et al. Cyclophosphamide, methotrexate and infusional 5-fluorouracil (infusional CMF) in metastatic breast cancer. Br. J. Cancer 77 , 1950-1956 (1998).

45 Palmieri C, Krell J, James C et al. Rechallenging with anthracyclines and taxanes in metastatic breast cancer. Nat. Rev. Clin. Oncol. 7, 561-574 (2010).

46 Geyer CE, Forster J, Lindquist D et al. Lapatinib plus capecitabine for HER2-positive advanced breast cancer. N. Engl. J. Med. 355, 2733-2743 (2006).

47 Baselga J, Cortés J, Kim S-B et al. Pertuzumab plus trastuzumab plus docetaxel for metastatic breast cancer. N. Engl. J. Med. 366, 109-119 (2012).

48 Child CG, Turcotte JG. Surgery and portal hypertension. In: The Liver and Portal Hypertension. CG Child (Ed.). Saunders, PA, USA, 50-64 (1964).

49 Pugh RN, Murray-Lyon IM, Dawson JL, Pietroni MC, Williams R. Transection of the oesophagus for bleeding oesophageal varices. Br. J. Surg. 60, 646-649 (1973).

50 Halaven $^{\circledR}$, package insert. Eisai, MA, USA. 